\title{
A mudança de decúbito na prevenção de lesão por pressão em pacientes na terapia intensiva
}

\begin{abstract}
RESUMO | O objetivo deste estudo consistiu em identificar os motivos que possam estar relacionados à dificuldade da realização da mudança de decúbito de pacientes em terapia intensiva. A metodologia empregada foi a pesquisa bibliográfica de artigos publicados entre 2013 e 2019, buscados na BVS de Enfermagem e no Google Acadêmico. De acordo com os estudos analisados, o Enfermeiro é o profissional responsável não apenas pelos cuidados assistenciais, mas pela avaliação dos fatores de risco de LP na admissão, da integridade da pele e dos resultados obtidos com as ações adotadas. A mudança de decúbito do paciente foi a medida mais adotada. Contudo, foram encontradas falhas na execução por falta de conhecimento, falha de integração entre os membros da equipe, número deficiente de pessoal ou inexistência de protocolo para a padronização das condutas dos profissionais de Enfermagem. Concluiu-se que é fundamental a participação do Enfermeiro nos programas educativos e a realização de ações voltadas para a educação dos membros de suas equipes para que também possam executar as medidas preventivas de forma correta e se tornarem promotores da saúde entre os familiares, cuidadores ou acompanhantes e a comunidade, transformando-os em co-participantes do processo de prevenção de LPs no paciente crítico.
\end{abstract}

Palavras-chaves: Lesão por Pressão; Terapia Intensiva; Prevenção.

ABSTRACT | The aim of this study was to identify the reasons that may be related to the difficulty of changing the position of patients in intensive care. The methodology used was the bibliographic search of articles published between 2013 and 2019, searched at the Nursing VHL and Google Scholar. According to the studies analyzed, the nurse is the professional responsible not only for the care provided, but for the assessment of the risk factors for Pl on admission, skin integrity and the results obtained with the actions taken. The patient's decubitus change was the most adopted measure. However, flaws in execution were found due to lack of knowledge, failure to integrate team members, deficient number of staff or no protocol for standardizing the conduct of nursing professionals. It was concluded that the participation of Nurses in the educational programs and the carrying out of actions aimed at the education of the members of their teams are essential so that they can also carry out preventive measures correctly and become health promoters among family members, caregivers or companions and the community, transforming them into co-participants in the process of preventing Pls in the critical patient.

Keywords: Pressure Injury; Intensive Care; Prevention.

RESUMEN | El objetivo de este estudio fue identificar las razones que pueden estar relacionadas con la dificultad de cambiar la posición de los pacientes en cuidados intensivos. La metodología utilizada fue la búsqueda bibliográfica de artículos publicados entre 2013 y 2019, buscados en Nursing VHL y Google Scholar. Según los estudios analizados, la enfermera es el profesional responsable no solo de la atención brindada, sino también de la evaluación de los factores de riesgo para PL al ingreso, integridad de la piel y los resultados obtenidos con las acciones tomadas. El cambio de decúbito del paciente fue la medida más adoptada. Sin embargo, se encontraron fallas en la ejecución debido a la falta de conocimiento, la falta de integración de los miembros del equipo, el número deficiente del personal o la falta de protocolo para estandarizar la conducta de los profesionales de enfermería. Se concluyó que la participación de las enfermeras en los programas educativos y la realización de acciones dirigidas a la educación de los miembros de sus equipos son esenciales para que también puedan llevar a cabo las medidas preventivas correctamente y convertirse en promotores de salud entre los familiares, cuidadores o compañeros y la comunidad, transformándolos en copartícipes en el proceso de prevención de LP en el paciente crítico.

Palavras claves: Lesión por Presión; Terapia Intensiva; Prevención.

\section{Adriely Duany Cardoso Gonçalves}

Enfermeira. Pós-graduada em Enfermagem em Alta Complexidade pela Universidade Veiga de Almeida. RJ/Brasil.

\section{Ana Lúcia Mota Binda}

Enfermeira. Pós-Graduada em Enfermagem em Alta Complexidade pela Universidade Veiga de Almeida. RJ/Brasil.

\section{Eriane Nascimento Pinto \\ Enfermeira. Doutora em Biociências.} Instituto Nacional do Câncer. RJ/Brasil.

\section{Elson Santos de Oliveira}

Enfermeiro. Doutorando em enfermagem e Biociências pela UNIRIO. Centro de Terapia Intensiva adulto do Instituto Nacional do Câncer e Centro de Terapia Intensiva COVID 5 do Hospital Universitário Pedro Ernesto-UERJ. Docente da Graduação de Enfermagem da Universidade Veiga de Almeida; RJ/Brasil.

\section{Isidoro Binda Netto}

Doutor em Ciências. Professor Associado da Disciplina de Anestesiologia da Universidade Federal do Estado do Rio de Janeiro (UNIRIO). RJ/Brasil.

\section{INTRODUÇÃO}

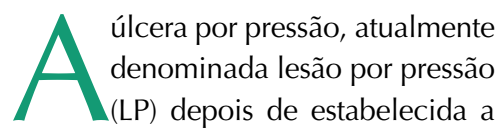
nova nomenclatura pelo National Pressure Ulcer Advisory Panel (NPUAP), em abril de $2016^{(1)}$, caracteriza-se por uma lesão tecidual causada pelo contato, durante longo período, das proeminências ósseas com superfícies rígidas, tais como colchões, cadeiras e macas que, por sua vez, resultarão em diminuição do fluxo sanguíneo provocando desnutrição da região comprimida, anóxia e necrose do tecido ${ }^{(2)}$.

A compressão da região corporal por

Recebido em: 13/05/2020

Aprovado em: 15/05/2020 
longo período causa um processo isquêmico, reduzindo o fluxo sanguíneo para os capilares que circundam o tecido e as adjacências e, consequentemente, dificultam ou impedem a chegada de oxigênio e nutrientes. Além da isquemia local, portanto, tem-se como resultado: a hipóxia, a formação de edema, o endurecimento da área afetada, a elevação da temperatura local decorrente da instalação de um processo inflamatório, o aparecimento de vermelhidão da pele ou eritema e, por fim, a apoptose ou morte celular e a necrose do tecido(3).

Embora a pressão sobre as regiões mais proeminentes do corpo ou com pouca quantidade de tecido subcutâneo - ex: calcâneos, quadris, maléolos, omoplatas e região sacra-coccigeana - contra uma superfície rígida seja considerado um importante fator de risco para o surgimento das LPs, também são apontadas as forças de cisalhamento, quando o paciente desliza do leito, deformando e destruindo o tecido; e a fricção, quando o paciente é frequentemente arrastado para movê-lo no leito ou retirá-lo do mesmo ${ }^{(3)}$.

De origem multicausal, as LPs se desenvolvem por fatores intrínsecos (edema, estado mental e nutricional, idade avançada, incontinência, inervação sensorial prejudicada, hidratação, hipotensão arterial, morbidades ou patologias associadas, motricidade involuntária excessiva, nível de consciência, perfusão tecidual reduzida, tonicidade muscular); ou extrínsecos ao paciente (pressão, fricção, cisalhamento, imobilização e umidade da pele). Assim, as ações preventivas requerem a participação de uma equipe multiprofissional composta por nutricionistas, fisioterapeutas, profissionais de Enfermagem entre outros ${ }^{(4,5)}$.

Diante de tais fatores, os pacientes com maior risco de desenvolver LPs são os desnutridos ao apresentarem ossos mais proeminentes; idosos pelo aumento da fragilidade da pele, pela redução da elasticidade e menor resistência às forças de tração e cisaIhamento; com doenças crônicas tais como diabetes mellitus e vasculares pelas alterações na circulação sanguínea e consequentemente, na perfusão tissular; osacamados em função de cirurgia de maior porte, traumas ou com deficiência física; ou que fazem uso de alguns anestésicos ou precisam ser sedados, alterando a percepção sensorial ${ }^{(6)}$.

A Escala de Braden compreende um importante instrumento de avaliação que analisa 6 fatores: percepção sensorial, umidade, atividade, mobilidade, nutrição e fricção e cisalhamento. Para cada fator, existem pontuações de 1 a 4 . A soma dos pontos determina a classificação de risco de LP do paciente, que varia de 4 a 23. Quanto maior a pontuação, menor o risco de LP. Assim, o paciente não apresenta risco quando a soma é de 19 a 23 pontos. Para o risco baixo, a soma varia de 15 a 18 pontos; moderado, de 13 a 14 pontos; alto, de 10 a 12 pontos; e muito alto, de 6 a 9 pontos. Embora seja útil, é preciso treinamento para a correta classificação de risco do paciente, já que pode se tornar subjetiva e interferir sobre as condutas adotadas pelos profissionais de Enfermagem ${ }^{(7)}$.

Há também a Escala de Waterlow, de maior utilização no Reino Unido. Os fatores considerados na avaliação são: estatura, peso, idade, sexo, apetite, continência, déficit neurológico, má nutrição tecidual, medicação em uso, mobilidade, tipo de pele, cirurgia de grande porte e trauma. O paciente é considerado de risco quando a pontuação é superior a 10. Quando acima de 15 e 20 pontos, considera- se o paciente como de alto risco e risco muito alto, respectivamente ${ }^{(8,9)}$.

As Unidades de Terapia Intensiva (UTIs) são unidades em que permanecem os pacientes considerados críticos, ou seja, aqueles com elevadas chances de falência ou instabilidade de diversos sistemas fisiológicos, necessitando, portanto, de controle minucioso e cuidados com mais frequência, juntamente com medidas terapêuticas mais complexas e/ou invasivas. Entre esses pacientes estão justamente os cirúrgicos, traumatizados ou com doenças que requerem a permanência do paciente no leito por mais tempo para recuperação da saúde, além dos fisicamente incapazes em decorrência da idade avançada ou por deficiência motora ${ }^{(7)}$.

Nas quatro UTIs de um hospital esta- dual de referência localizado em Natal, Rio Grande do Norte, a prevalência de LPs variou de $44,4 \%$ a $87,5 \%$, resultando em uma taxa média de $69 \%{ }^{(10)}$. Em uma UTI Adulto em Campinas, São Paulo, a incidência foi de $34,78 \%$, percentual também considerado elevado. Ademais, houve pacientes que desenvolveram mais de uma lesão ${ }^{(11)}$.

No entanto, as LPs são complicações passíveis de prevenção. Por isso, o desenvolvimento de LPs nas instituições de saúde vem sendo interpretado como um importante fator de indicação da qualidade dos serviços prestados ${ }^{(4)}$. Os indicadores de qualidade permitem o monitoramento de atividades identificando quais os assuntos específicos dos resultados alcançados e os esperados, dentro de uma organização de saúde, que devem ser motivo de uma revisão para a meIhoria da qualidade. Os indicadores podem estar relacionados à estrutura, ao processo ou aos resultados ${ }^{(12,13)}$.

A estrutura trata de características mais estáveis, incluindo informações sobre recursos materiais (como instalações e equipamentos), humanos (número e qualificação) e estrutura organizacional (critérios de operação, sistemas de avaliação, protocolos entre outros). Quando se trata de processo, objetiva-se analisar como os profissionais de saúde realizam suas atividades, atuam na condução dos cuidados e na interação com os pacientes. Enfim, o resultado volta-se ao estudo dos efeitos e consequências das intervenções, utilizando taxas, indicadores, parâmetros de saúde e de satisfação da clientela ${ }^{(13)}$.

A prevenção das LPs pode ser alcançada através de diferentes estratégias adotadas pelos Enfermeiros, tais como: aporte nutricional; tratamento tópico; controle da umidade da pele; e a mudança de decúbito ou decúbito de execução relativamente simples e que não implica gastos hospitalares, tornando conveniente a sua adoção como parte da rotina de cuidados ao longo do período de internação do paciente ${ }^{(14)}$.

Diversas tecnologias vêm sendo criadas para reduzir o risco de desenvolvimento dessas lesões. O uso de colchão de alternância de ar, coxim viscoelásticos, 
protocolos de prevenção são exemplos de algumas das tecnologias leves e duras empregadas pela Enfermagem. A literatura recomenda a mudança de decúbito do paciente com o intervalo mínimo a cada duas horas. Porém no cotidiano vivenciado pelos autores esse intervalo não é realizado por motivos diversos, por exemplo, piora da instabilidade hemodinâmica, contenção mecânica, falha da assistência de Enfermagem entre outros. Embora existam recursos tecnológicos que aliviam a pressão principalmente sobre as proeminências ósseas, a mudança de decúbito é considerada de grande importância e eficácia, mas também uma medida totalmente dependente da ação humana ${ }^{(15,16)}$.

A execução da mudança de decúbito pode ser influenciada por fatores do próprio paciente (atelectasias, pós-operatório de cirurgias plásticas, uso de aminas vasoativas, delírio entre outros), da estrutura (tipos de colchões disponíveis, carga de trabalho, qualificação profissional, protocolos assistenciais entre outros) e do processo (aplicabilidade das escalas de avaliação de risco, adaptações feitas para a implementação das medidas de prevenção). Entende-se que esses fatores refletem os indicadores de resultado, especialmente na incidência de LPs na região sacra, causada pela necessidade ou não do paciente permanecer na maior parte do tempo em decúbito dorsal|(16).

O Enfermeiro tem papel de destaque no gerenciamento do cuidado, pois é o responsável pela avaliação do risco e a partir deste estabelecer as medidas de prevenção que deverão ser empregadas, bem como a supervisão dos cuidados prestados. Logo, é o profissional de saúde que mais está em contato com o paciente, prestando serviço praticamente 24 horas por $\mathrm{dia}^{(4)}$.

Diante desta problemática, o objeto deste estudo é a aplicabilidade da mudança de decúbito pela Enfermagem na terapia intensiva. Já a questão norteadora é: Quais os desafios enfrentados pela Enfermagem na aplicabilidade da mudança de decúbito na terapia intensiva? Desta forma, o estudo tem como objetivo principal identificar os motivos que possam estar relacionados à dificuldade da realização da mudança de decúbito de pacientes em terapia intensiva.

\section{METODOLOGIA}

A metodologia adotada foi a do tipo revisão integrativa, permitindo a análise do conhecimento e de seus efeitos na prática $^{(17)}$. A revisão ainda foi feita adotando o sistema duplo-cego para a busca dos artigos. Assim, o processo de seleção, inclusão e exclusão dos artigos realizado pelo autor foram confrontados com os achados do revisor para validação dessa busca. Quanto à abordagem, o estudo se caracteriza como qualitativo.

A coleta de dados foi realizada no período de outubro de 2018 e julho de 2019. O problema a ser investigado é: Quais os desafios enfrentados pela Enfermagem na aplicabilidade da mudança de decúbito na terapia intensiva?

O questionamento central ou problema de pesquisa surgiu mediante a descrição dos componentes que apresentam relação com a problemática da elevada incidência de lesão por pressão em pacientes internados em terapia intensiva. Portanto, tem-se para as iniciais: $\mathrm{P}$ - Pacientes internados em terapia intensiva, considerados de risco ou não de desenvolvimento de lesões por pressão, possuindo ou não lesão por pres- são no momento da admissão hospitalar ou no início do estudo; I - Mudança de decúbito pelos profissionais de Enfermagem para a prevenção de lesão por pressão nos pacientes críticos; C - Comparação entre a frequência da mudança de decúbito executadas ou reportadas pelos profissionais de Enfermagem, verificando a diferença e similaridade entre elas e seus principais motivos; O - Verificação do conhecimento sobre a mudança de decúbito adequada ou de sua adoção em concordância com a literatura para prevenção das lesões por pressão ou a redução de sua incidência nos pacientes em terapia intensiva.

Os critérios de inclusão estabelcidos foram: disponível na íntegra gratuitamente; idiomas português ou inglês; estudos de campo ou caso; estudos envolvendo seres humanos; e recorte temporal de 2013 a 2018-2019 (últimos 5 anos). Já os critérios de exclusão foram: estudos publicados anteriormente a 2013; estudos publicados em outro idioma; estudos de revisão literária; estudos in vitro e envolvendo animais.

A busca foi realizada através da Biblioteca Virtual em Saúde (BVS) de Enfermagem. O fluxograma, a seguir (Figura 1), demonstra os passos efetuados para a seleção dos artigos buscados na BVS, adotando os critérios de inclusão e exclusão anteriormente descritos.
Figura 1. Fluxograma de busca e seleção dos estudos na BVS. Rio de Janeiro, RJ, Brasil, 2018-2019

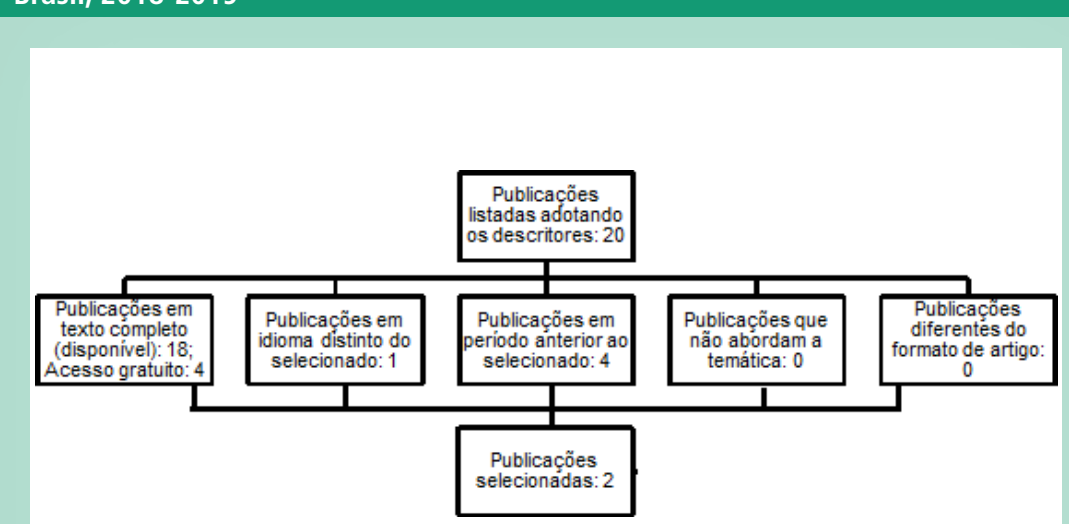


Diante desses achados, decidiu-se realizar semelhante busca no Google Scholar para aumentar o número de artigos selecionados. Os mesmos descritores e critérios de inclusão e exclusão foram adotados. Os artigos publicados em revistas científicas eram selecionados na ordem em que apareciam para verificação da conformidade com o tema, considerando uma lista de 20 publicações.

Portanto, a análise dos resultados foi feita com base em 13 artigos, sendo 11
Figura 2. Fluxograma de busca e seleção dos estudos no Google Scholar. Rio de Janeiro, RJ, Brasil, 2018-2019

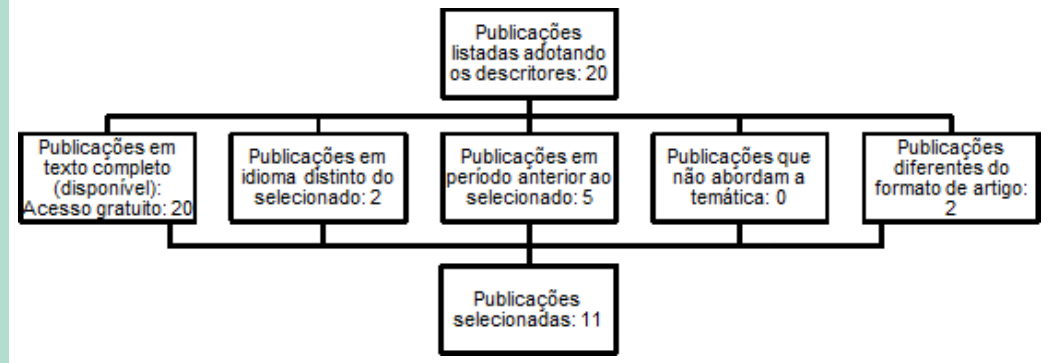

em português (Google Scholar) e 02 em inglês (BVS).

A análise dos dados coletados foi feita através da leitura dos resumos, objetivos, métodos e dos principais resultados para a verificação de possíveis convergências e divergências entre os autores em relação à prática da mudança de decúbito do paciente pelos Enfermeiros para prevenção de lesão por pressão nos pacientes críticos, além dos efeitos sobre a ocorrência dessas lesões, quando os dados estavam disponíveis, a fim de possibilitar a discussão dos resultados e a comparação com o preconizado pela literatura.

\section{RESULTADOS}

Foram revisados 13 estudos de caso ou campo buscados na BVS $(\mathrm{N}=02)$ de Enfermagem e no Google Scholar $(\mathrm{N}=11)$. Os principais achados de cada estudo estão descritos no Quadro 1 , a seguir.

Quadro 1. Principais achados nos artigos selecionados. Rio de Janeiro, RJ, Brasil, 2018-2019

\begin{tabular}{|c|c|c|c|}
\hline Autor(es) (ano) & Assunto/ Amostra & Resultados & Conclusões \\
\hline $\begin{array}{l}\text { Swafford, Culpepper e } \\
\text { Dunn (2016 }\end{array}$ & $\begin{array}{l}\text { Eficácia de um programa de pre- } \\
\text { venção de LPs adquiridas em hospi- } \\
\text { tal por em uma UTI adulta, visando } \\
\text { a redução de pelo menos } 50 \% \text { em } \\
12 \text { meses. } \\
\text { Componentes do programa: escala } \\
\text { de Braden, protoco-lo revisado } \\
\text { de cuidados com a pele, reposicio- } \\
\text { nadores fluidizados e cura- tivos } \\
\text { com gel de silicone. }\end{array}$ & $\begin{array}{l}\text { A incidência de LPs foi reduzida } \\
\text { em } 69 \% \text {. } 0 \text { potencial de redução } \\
\text { de custos resultante dessa dimi- } \\
\text { nuição da incidência foi de apro- } \\
\text { ximadamente } 1 \text { milhão de dóla- } \\
\text { res dentro do período avaliado. }\end{array}$ & $\begin{array}{l}0 \text { programa de prevenção de LP ado- } \\
\text { tado se mostrou eficaz na redução da } \\
\text { incidência de LPs na UTI investigada. } \\
\text { A educação e motivação da equipe e o } \\
\text { incentivo à proatividade dos Enfermei- } \\
\text { ros foram fundamentais para a detec- } \\
\text { ção dos pacientes com risco de LP e a } \\
\text { adoção do protocolo. }\end{array}$ \\
\hline Davis e Kotowski (2015) & $\begin{array}{l}\text { Influência do design de } 4 \text { leitos } \\
\text { sobre fricção / deslizamento e com- } \\
\text { pressão do tronco. } \\
\mathrm{N}=12 \text { indivíduos saudáveis simu- } \\
\text { lando pacientes em coma. }\end{array}$ & $\begin{array}{l}0 \text { deslizamento do corpo do pa- } \\
\text { ciente no leito e a compressão } \\
\text { sobre o tronco foi significativa- } \\
\text { mente maior na elevação da ca- } \\
\text { beceira a } 30^{\circ} \text { e } 45^{\circ} \text { e no retorno a } \\
0^{\circ} \text { em } 3 \text { modelos de leito, exceto } \\
\text { naquele com um mecanismo na } \\
\text { região da cabeça e do tronco } \\
\text { que se alongava junto com o le- } \\
\text { vantamento da cabeceira e um } \\
\text { contorno automático do joelho } \\
\text { que também se levantava com a } \\
\text { cabeceira. }\end{array}$ & $\begin{array}{l}0 \text { design da cama afetoua quantida- } \\
\text { de de deslizamento e compressão do } \\
\text { tronco, sendo maiores nos leitos com } \\
\text { desenhos de pivô de secção de cabeça } \\
\text { simples, que não têm a capacida- de } \\
\text { de deslizar para trás ou se alongar, au- } \\
\text { mentando o risco de LPs. }\end{array}$ \\
\hline
\end{tabular}




\begin{tabular}{|c|c|c|c|}
\hline $\begin{array}{l}\text { Mendonça } \\
\text { et al. (2018) }\end{array}$ & $\begin{array}{l}\text { Ações de enfermagem para pre- } \\
\text { venção de LP e sua ocorrência } \\
\text { em CTIs. } \\
\mathrm{N}=104 \text { enfermeiros de } 2 \text { hospi- } \\
\text { tais de ensino de Campo Grande, } \\
\text { Mato Grosso do Sul }\end{array}$ & $\begin{array}{l}\text { A ocorrência média de LP era de } 49 \% \text { nos } 2 \text { hospitais. } \\
\text { Ações preventivas: inspeção da pele, aplicação de } \\
\text { cobertura hidrocoloide em região sacral, higieniza- } \\
\text { ção externa, troca de fixação do cateter orotraque- } \\
\text { al e/ou cateter nasoenteral, elevação da cabeceira } \\
\text { a } 30^{\circ} \text { e mudança de posicionamento a cada } 2 \text { ho- } \\
\text { ras (a mais praticada e fortemente relacionada à } \\
\text { ausência da LP). }\end{array}$ & $\begin{array}{l}\text { A elaboração e implementação de } \\
\text { protocolos, o acompanhamento dos } \\
\text { registros e dos grupos de maior risco } \\
\text { orientaram a prescri ção das ações } \\
\text { preventivas adequadas para LP. }\end{array}$ \\
\hline $\begin{array}{l}\text { Sanches et } \\
\text { al. (2018) }\end{array}$ & $\begin{array}{l}\text { Adesão da equipe de Enferma- } \\
\text { gem ao protocolo de LP e segu- } \\
\text { rança do paciente em UTIs. } \\
\mathrm{N}=945 \text { pacientes com } 18 \text { anos } \\
\text { ou mais internados em } 6 \text { UTIs do } \\
\text { Hospital de Base de São José do } \\
\text { Rio Preto, São Paulo. }\end{array}$ & $\begin{array}{l}\text { LP presente empenas } 5,29 \% \text { dos pacientes duran- } \\
\text { te a internação conforme checklist à beira do leito; } \\
0 \text { avanço da idade foi tido como variável depen- } \\
\text { dente do desen- volvimento de LP e sua maior } \\
\text { incidência; } \\
\text { A mudança de decúbito não foi correlacionada } \\
\text { com o aumento das LPs, confirmando a eficácia } \\
\text { da medida preventiva e a boa adesão pelos pro- } \\
\text { fissio- nais de Enfermagem. As UTls possuem o } \\
\text { relógio de mudança de decúbito, indicando em } \\
\text { qual posição o paciente deve estar naquela de- } \\
\text { terminada hora do dia. }\end{array}$ & $\begin{array}{l}\text { Constatou-se a adesão da equipe de } \\
\text { Enfermagem ao protocolo pelo baixo ín- } \\
\text { dice de LP em relação a outros estudos. } \\
\text { A associação apenas com a idade refle- } \\
\text { te a segurança dos pacientes quanto à } \\
\text { medida de prevenção da LP (mudança } \\
\text { de decúbito) e sua eficácia pela baixa } \\
\text { incidência. }\end{array}$ \\
\hline $\begin{array}{l}\text { Vasconcelos } \\
\text { e Caliri } \\
\text { (2017) }\end{array}$ & $\begin{array}{l}\text { Ações dos profissionais de Enfer- } \\
\text { magem, antes e após utilização } \\
\text { de protocolo de prevenção de } \\
\text { LP em UTI hospital de ensino em } \\
\text { João Pessoa, Paraíba. }\end{array}$ & $\begin{array}{l}\text { Após uso do protocolo, observou-se maior frequ- } \\
\text { ência das açães: } \\
\text { - Avaliação do risco para LP na admissão e nos } \\
\text { dias subsequentes; } \\
\text { - Observação e proteção de proeminências ósse- } \\
\text { as e aplicação de hidratante; } \\
\text { - Mudança de decúbito. A frequência não foi des- } \\
\text { crita, mas foi baseada na condição clínica do pa- } \\
\text { ciente, respeitando ainda a hemodinâmica para } \\
\text { elevação da cabeceira entre } 30^{\circ} \text { e } 45^{\circ} \text { e evitar } \\
\text { pneumonia asso-ciada à ventilação mecânica. } \\
\text { Mas houve ausência de registro em } 15 \% \text { dos pron- } \\
\text { tuários, dificultando a comunicação entre os pro- } \\
\text { fissionais sobre a integridade da pele. }\end{array}$ & $\begin{array}{l}\text { Houveaumento na frequência de ações } \\
\text { preventivas após uso do protocolo. A } \\
\text { ferramenta é essencial para adoção } \\
\text { das recomendações baseadas em evi- } \\
\text { dências científicas pelos profissionais. }\end{array}$ \\
\hline $\begin{array}{l}\text { Olkoski e } \\
\text { Assis (2016) }\end{array}$ & $\begin{array}{l}\text { Efetividade de uma campanha } \\
\text { para prevenção de LP em um } \\
\text { hospital de ensino de Curitiba. }\end{array}$ & $\begin{array}{l}\text { mudança de decúbito a cada } 3 \text { horas; lateraliza- } \\
\text { ção com angulação menor que } 90^{\circ} \text {; elevaçãao de } \\
\text { cabeceira abaixo de } 45^{\circ} \text {; elevação de calcâneos } \\
\text { com apoio sob as panturrilhas. } \\
\text { Itens com queda ou que merecem atenção: ore- } \\
\text { Ihas livres de pressão, fixação adequada de cate- } \\
\text { teres e drenos. } \\
\text { Itens com pequeno aumento da adesão: uso de } \\
\text { travesseiros de espuma sob a cabeça e de col- } \\
\text { chões especiais. }\end{array}$ & $\begin{array}{l}\text { A campanha foi considerada efetiva, } \\
\text { porém é necessária uma abordagem } \\
\text { contínua e sistemática para o aumento } \\
\text { da adesão em outras medidas preventi- } \\
\text { vas e de auxílio à prevenção. }\end{array}$ \\
\hline $\begin{array}{l}\text { Pereira, } \\
\text { Ludvich e } \\
\text { Omizzolo } \\
\text { (2016) }\end{array}$ & $\begin{array}{l}\text { Ações de Enfermagem em uma } \\
\text { UTI de hospital da serra catari- } \\
\text { nense; } \\
\mathrm{N}=12 \\
\text { profissionais de Enfermagem; } \\
\text { Categorias de análise: } \\
\text { - Conhecimento profissional; } \\
\text { - Classificação de risco dos pacientes; } \\
\text { - Notificação das LPs. }\end{array}$ & $\begin{array}{l}\text { - Conhecimento profissional: mudança de decúbi- } \\
\text { to a cada } 2 \text { horas (principal ação realizada), uso de } \\
\text { colchão pneumático e de hidratante (hidrocoloide, } \\
\text { ácido graxo, óleo de girassol), uso de coxins; } \\
\text { - Classificação de risco: escala de Braden (na in- } \\
\text { ternação) e colagem de adesivo colorido nos pés } \\
\text { da cama; } \\
\text { - Notificação de LPs: muitos profissionais (auxi- } \\
\text { liar e técnico de Enfermagem, principalmente) } \\
\text { não conheciam o formulário para notificação de } \\
\text { eventos adversos. }\end{array}$ & $\begin{array}{l}\text { A alta prevalência e incidência de LP. } \\
\text { Necessidade de uso da Escala de Bra- } \\
\text { den no momento da admissão e escla- } \\
\text { recimento quanto ao preenchimento } \\
\text { do formulário por todos da equipe e } \\
\text { não somente pelo Enfermeiro. }\end{array}$ \\
\hline
\end{tabular}




\begin{tabular}{|c|c|c|c|}
\hline $\begin{array}{l}\text { Cruz et al. } \\
\text { (2015) }\end{array}$ & $\begin{array}{l}\text { Percepção de Enfermeiros quanto } \\
\text { à prevenção de LPs em um Hos- } \\
\text { pital Universitário em Petrolina, } \\
\text { Pernambuco. } \\
\mathrm{N}=8 \text { Enfermeiros. }\end{array}$ & $\begin{array}{l}\text { A maioria desconhecia a existência de um pro- } \\
\text { tocolo de prevenção no hospital ou quando era } \\
\text { conhecido, as medidas preventivas não eram } \\
\text { adotadas conforme o documento. } \\
\text { A mudança de decúbito era adotada por todos } \\
\text { os profissionais, mas somente um afirmou exe- } \\
\text { cutá-la a cada } 2 \text { horas. Outro relatou cobrar a } \\
\text { ajuda do técnico de Enfermagem para o respeito } \\
\text { aos intervalos. } \\
\text { Todavia, houve falta de padronização e sistema- } \\
\text { tização das medidas preventivas adotadas devi- } \\
\text { do ao conhecimento } \\
\text { diferen-te entre eles. Uns adotavam o protocolo, } \\
\text { outros colocavam em prática o que foi aprendido } \\
\text { no curso de graduação ou em outros ofertados } \\
\text { por empresasfabricantes de curativos. }\end{array}$ & $\begin{array}{l}\text { Necessidade de disseminação do co- } \\
\text { nhecimento sobre a existência de pro- } \\
\text { tocolo de prevenção e sistematização } \\
\text { das ações. }\end{array}$ \\
\hline $\begin{array}{l}\text { Mattos et } \\
\text { al. (2015) }\end{array}$ & $\begin{array}{l}\text { Ações de prevenção de LP em } \\
\text { UTI e impactos da educação de } \\
\text { profissionais de Enfermagem e } \\
\text { acompanhantes acerca das medi- } \\
\text { das preventivas. }\end{array}$ & $\begin{array}{l}\text { No pré-teste (antes das intervenções educativas), } \\
\text { as ações preventivas eram executadas principal- } \\
\text { mente pelos acompanhantes }(97,06 \%) \text {, seguido } \\
\text { de técnicos de Enfermagem }(57,9 \%) \text { e Enfermei- } \\
\text { ros ( } 33,33 \%) \text {. } \\
\text { A mudança de decúbito era a principal medida } \\
\text { adotada, mas sem periodicidade. } \\
\text { Auxiliares de prevenção corretos eram usados } \\
\text { como travesseiros e coxins. Mas também aqueles } \\
\text { considerados } \\
\text { inadequados (boias de plástico, luva d'água, col- } \\
\text { chões plásticos de água e de ar não pneumáticos). } \\
\text { No pós-teste houve aumento significativo dos } \\
\text { acertos relacionados às perguntas sobre preven- } \\
\text { ção, mas as técnicas errôneas de prevenção ainda } \\
\text { eram adotadas, pois de } 180 \text { profissionais de saú- } \\
\text { de, somente } 46 \text { participaram de todas as palestras } \\
\text { educativas. }\end{array}$ & $\begin{array}{l}\text { Necessidade de ações educativas volta- } \\
\text { das para a uniformização do conheci- } \\
\text { mento sobre prevenção de LP e adoção } \\
\text { das medidas preventivas corretamente } \\
\text { e de forma sistematizada }\end{array}$ \\
\hline $\begin{array}{l}\text { Albuquer- } \\
\text { que et al. } \\
\text { (2014) }\end{array}$ & $\begin{array}{l}\text { Conhecimento sobre prevenção } \\
\text { de LP em CTIs de } 4 \text { hospitais de } \\
\text { João Pessoa, Paraíba. } \\
\mathrm{N}=40 \text { Enfermei- ros. }\end{array}$ & $\begin{array}{l}\text { As respostas certas sobre prevenção variaram de } \\
25 \% \text { a } 100 \% \text {. } \\
\text { Itens com menos acertos (abaixo de } 50 \% \text { ): re- } \\
\text { posicionamento do paciente sentado a cada } 2 \\
\text { horas ou do paciente imóvel no leito a cada } 3 \\
\text { horas; rodas d'água não auxiliam na prevenção; } \\
\text { imobilidade, incontinência, nutrição inadequada } \\
\text { e alteração do nível de consciência como fato- } \\
\text { res de risco; protetores de calcâneos não são } \\
\text { recomendados; não massagear áreas de proemi- } \\
\text { nência ou hiperemiadas; não elevar a cabeceira } \\
\text { acima de } 30^{\circ} \text {. } \\
\text { Itens com mais acertos ( } 100 \% \text { ): avaliação de } \\
\text { risco de LP na admissão; mudança de decúbito } \\
\text { em intervalos determinados para cada paciente; } \\
\text { mobilização e transferência do paciente feita por } \\
\text { duas ou mais pessoas; proeminências ósseas não } \\
\text { devem estar em contato entre si. }\end{array}$ & $\begin{array}{l}\text { Discrepância entre conhecimentos so- } \\
\text { bre prevenção e as ações realizadas. }\end{array}$ \\
\hline
\end{tabular}




\begin{tabular}{|c|c|c|c|}
\hline $\begin{array}{l}\text { Oliveira, } \\
\text { Santos e } \\
\text { Almeida } \\
\text { (2014 }\end{array}$ & $\begin{array}{l}\text { Ações de Enfermagem em uma } \\
\text { UTI de Hospital Público de Mon- } \\
\text { tes Claros, Minas Gerais. } \\
\mathrm{N}=22 \\
\text { profissionais de Enfermagem. }\end{array}$ & $\begin{array}{l}\text { Ações preventivas realizadas pela equipe de En- } \\
\text { fermagem: } \\
100 \% \text { - mudança de decúbito a cada } 2 \text { ou } 3 \text { ho- } \\
\text { ras, uso de colchão especial, de curativos para } \\
\text { proteger proeminências ósseas, uso de hidratan- } \\
\text { tes na pele seca ou em áreas ressecadas, verifica- } \\
\text { ção de áreas susceptíveis da pele dos pacientes } \\
\text { em risco de desenvolver LP, controle de umidade } \\
\text { no leito, roupas de cama bem esticadas, calcâne- } \\
\text { os livres de pressão com auxílio de travesseiro na } \\
\text { região posterior da perna; } \\
72,72 \% \text { - massagem nas áreas de proeminência } \\
\text { ou hiperemiadas; } \\
68,19 \% \text { - elevação da cabeceira (desses, 13,33\% } \\
\text { a realiza corretamente); } \\
63,64 \% \text { - uso de quadro demonstrativo de áreas } \\
\text { de risco de LP ( } 36,36 \% \text { não a usam ou conhecem). }\end{array}$ & $\begin{array}{l}\text { Necessidade de implantação de um } \\
\text { protocolo de prevenção de LPs. }\end{array}$ \\
\hline
\end{tabular}

\section{DISCUSSÃO}

As LPs atingem principalmente os pacientes com a mobilidade prejudicada e que passam por longo período de internação, a exemplo daqueles em terapia intensiva. Porém, as lesões são passíveis de prevenção. Para isso, as ações do Enfermeiro são fundamentais, pois é o profissional de saúde que se encontra mais tempo à beira do leito do paciente, cabendo a ele a avaliação do risco de LP na admissão do paciente, a avaliação diária e sistemática dos fatores de risco e também da pele e os cuidados assistenciais e de avaliação das medidas adotadas para sua continuidade ou alteração com vistas a garantir a eficácia preventiva e a redução da incidência das lesões nos hospitais ${ }^{(5,18)}$.
Para avaliação do risco de LP, a escala de Braden está entre os instrumentos mais utilizados no campo da Enfermagem. Um dos estudos analisados revelou seu uso somente quando o paciente havia sido internado ${ }^{(19)}$. A literatura preconiza seu emprego no momento da $\operatorname{admissão~}^{(5,7)}$. Para que as ações sejam precoce e adequadamente definidas, planejadas e disseminadas entre os profissionais de Enfermagem, contribuindo para sua execução correta e a redução do risco de desenvolvimento ou da incidência das LPs, como constatado por autores $^{(20-22)}$.

Também foi possível constatar que as campanhas, os programas ou protocolos de prevenção desenvolvidos e implementados exerceram influência positiva sobre os profissionais de Enfermagem por permitir o conhecimento sobre os pacientes internados (ex: risco de LP), a execução correta das medidas preventivas, tendo como resultado a redução da incidência de $\operatorname{LPS}^{(2,20,21,23-25)}$.

Todavia, os protocolos precisam ser revisados e implementados de forma contínua e permanente para que as informações sobre as medidas preventivas cheguem a todos os profissionais de Enfermagem, já que foram apontados problemas como a baixa ou não adesão ao protocolo de prevenção por desconhecimento da existência do mesmo, além de falta de padronização e uniformização na execução das medidas ${ }^{(2,5,14,22,26-28)}$. Autores $^{(14)}$ ainda mencionaram como agravante a não participação dos programas 
educativos ofertados por boa parte dos profissionais embora tivessem contemplado todos os horários e esquemas de plantão institucional. Por isso, também foi ressaltada a importância da motivação da equipe e o incentivo à proatividade por estudo ${ }^{(21)}$.

No que se refere à mudança de decúbito, a grande maioria dos estudos analisados apontou essa medida de prevenção como a mais executada ${ }^{(5,14,19,20,22,23,26,27-29)}$, além de ter sido a mais relacionada com a ausência de LP( ${ }^{23)}$.

Essa medida tem por objetivo interromper a pressão incidida sobre a pele do paciente, possibilitando a chegada de sangue essencial à oxigenação e nutrição dos tecidos. Ademais, pesquisadores afirmaram que somente o uso de materiais auxiliares ou outra medida preventiva isoladamente não previne o aparecimento da lesão. Por isso, é dada tanta importância à mudança de decúbito ${ }^{(4,18,30)}$.

No entanto, é fundamental que a mudança de decúbito seja executada a cada 2 ou 3 horas ou em intervalos estabelecidos conforme o estado de saúde do paciente, incluindo nível de atividade e mobilidade, condição médica geral e da pele, tolerância tecidual(19,20,23,24,29,5), o que não foi verificado em outros estu$\operatorname{dos}^{(14,22,26-28)}$.

Nesse sentido, convém salientar que autores $^{(14)}$, ao destacarem o uso de relógio de mudança de decúbito, para que os intervalos fossem respeitados. O relógio compreende um impresso plastificado fixado na parede, acima da cabeceira da cama. Cores diferentes são usadas para distinguir as horas em que o paciente deve ser mudado de posição.

A elevação da cabeceira também precisa ser observada durante a mudança de decúbito do paciente e compreendida como parte dessa medida. Diversos pesquisadores recomendaram o ângulo $30^{\circ}$ para não haver o deslizamento do corpo do paciente sobre a superfície do leito e a ocorrência de atrito e cisalhamento que podem danificar a integridade da pele, principalmente nas regiões das proeminências ósseas como sacrococcigiana $^{(5,22,27)}$.

No entanto, foi apontada a possibilidade de levantamento em até $45^{\circ}$ conforme a hemodinâmica do paciente a fim de evitar pneumonia associada à ventilação mecânica(20) ou de acordo com o modelo de cama, com mecanismo de alongamento da região da cabeça e do tronco; e de elevação do contorno automático da parte posterior do joelho que acompanham o levantamento da cabeceira. O deslizamento no leito não somente eleva o risco de LP entre os pacientes pelos danos causados à pele, mas para os cuidadores/familiares ou os profissionais de Enfermagem devido ao aumento desnecessário da frequência de mudança de decúbito, causando dores e injúrias à coluna vertebral, principalmente à região lombar ${ }^{(31)}$.

Também não se deve ignorar a presença de obstáculos à execução das medidas preventivas, tais como: a sobrecarga de trabalho pelo número deficiente de profissionais; carência de materiais auxiliares; baixa adesão da equipe; e, obviamente, o déficit de conhecimento e de programas de prevenção padronizado e sistemático, ou a sua existência pouco divulgada entre os profissionais de Enfermagem para a uniformização dos saberes e sua execução na prática, facilitando a organização do trabalho e distribuição das ações de acordo com uma ordem de prioridade considerando o número de pessoal existente em cada plantão $0^{(14,26-28)}$.

Uma estratégia que ajudou a organização do trabalho das equipes das UTIs de um Hospital de Base de São José do Rio Preto, São Paulo, foi o uso do relógio de mudança de decúbito para indicar em qual posição o paciente deveria estar em determinada hora do dia. O relógio era um impresso plastificado fixado na parede logo acima cama, servindo como lembrete de fácil visualização. Cores diferentes foram adotadas para cada horário da mudança, contribuindo para a identificação da hora em que o paciente deveria ser reposiciona- 
do conforme o protocolo. A eficácia da medida preventiva foi confirmada mediante a baixa incidência de LP entre os pacientes durante a internação - 5,29\%; $\mathrm{N}=50$ de $945^{(24)}$.

Outra questão que merece destaque é a carência de registros dos efeitos obtidos com as ações executadas para notificação de LPs pelos profissionais, principalmente auxiliares e técnicos de Enfermagem, resultando em elevada incidência e prevalência das lesões na UTI do hospital investigado(19). Segundo autores $^{(5)}$, o registro das condutas de Enfermagem e das respostas do paciente é primordial para que o planejamento eficaz das ações futuras.

Ademais, cabe ressaltar a importância da integração entre Enfermeiros e familiares/cuidadores ou acompanhantes, já que estes constituíam a grande maioria dos sujeitos que executavam as medidas preventivas em uma UTI ${ }^{(14)}$. Contudo, as ações eram realizadas de forma incorreta ou insuficiente. Por isso, as ações socioeducativas também devem fazer parte do planejamento estratégico dos Enfermeiros para a prevenção de LPs.

O mesmo vale para as ações de educação voltadas às equipes para que as tarefas possam ser distribuídas entre os técnicos e auxiliares de Enfermagem, por exemplo, contribuindo para o alívio da sobrecarga de tarefas do Enfermeiro e a garantia de execução das medidas em conformidade com as normas e os intervalos de tempo estabelecidos ${ }^{(5)}$, já que somente um Enfermeiro buscou apoio do técnico de Enfermagem para executar a mudança de decúbito nos intervalos corretos conforme o estudo(26).

Ao final, os profissionais das equipes de Enfermagem também estariam preparados para disseminar o conhecimento, tornando os familiares/cuidadores ou acompanhantes co-participantes do processo de prevenção da LP através de instruções e orientações. Infelizmente, esse cenário não foi observado em diversos estudos analisados, ao revelarem o despreparo de parte dos profissionais de
Enfermagem, fazendo com que medidas de prevenção errôneas ainda fossem exe-

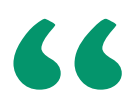

Ademais, cabe ressaltar a importância da integração entre Enfermeiros e familiares/ cuidadores ou acompanhantes, já que estes constituíam a grande maioria dos sujeitos que executavam as medidas preventivas em uma UTI

cutadas ou consideradas corretas - ex: massagem nas proeminências ou áreas hiperemiadas, uso de boias de plástico, luvas d'água, colchões plásticos de água e de ar não pneumáticos ${ }^{(2,5,14,22,26)}$.

\section{CONCLUSÃO}

Com base nesta revisão de literatura, foi possível concluir que o Enfermeiro desempenha um papel fundamental para a prevenção da LP no paciente em terapia intensiva, pois a ele cabe a avaliação dos fatores de risco no momento da admissão, inspeção da pele diariamente, além de outras ações assistenciais, como controle da umidade, hidratação da pele e oral, nutrição adequada e alívio da pressão sobre as proeminências ósseas através do uso de materiais auxiliares, da elevação da cabeceira e mudança de decúbito.

A mudança de decúbito, por sua vez, é uma medida que não implica custos, mas depende, e muito, dos profissionais de Enfermagem para sua execução correta e em intervalos regulares. Essa medida ainda revelou ser a mais adotada pelos Enfermeiros. Porém, sua execução deve ser feita respeitando a condição clínica do paciente e a cada 3 horas, no máximo, para que seja eficiente.

Contudo, é preciso que gestores reconheçam e valorizem a atuação dos profissionais de Enfermagem no sentido de dar-lhes condições de trabalho adequadas para que possam atender as necessidades da demanda; além de planejar a contratação de mais profissionais quando necessário. Ou seja, os gestores devem ter em mente que ao atuarem na prevenção, nos cuidados e no acompanhamento da saúde individual e coletiva, os profissionais de Enfermagem também contribuem para uma importante redução da incidência e prevalência da LP nos hospitais brasileiros e dos gastos públicos com a saúde.

A Enfermagem pode ser considerada a área que mais contribui para a prevenção de LPs já que os profissionais são aqueles que mais estão em contato com os pacientes. No entanto, os estudos 
aqui analisados também revelaram como obstáculos à prevenção da LP o conhecimento insuficiente sobre as medidas ou sua execução errônea pelos profissionais de Enfermagem. Por esta razão, é conveniente o incentivo à adoção de um protocolo de ações preventivas padronizadas. A padronização favorece a adoção das medidas sem desencontros de informações ou confusões entre os profissionais de Enfermagem.

Mas para assegurar o entendimento dos profissionais de Enfermagem acerca das medidas preventivas e do protocolo implementado para a execução correta das ações, convém a participação do Enfermeiro nos programas educativos e a realização de ações educacionais e de monitoramento/avaliação contínua do trabalho das equipes. Por isso, a educação também deve integrar o planejamento para uniformizar as condutas dos profissionais e, logo, reduzir o risco de ocorrência de LPs, além de melhorar o trabalho das equipes, tornando-o fluido e mais organizado. Por sua vez, os profissionais das equipes de Enfermagem estarão aptos a atuar como verdadeiros promotores da saúde através da educação de familiares, cuidadores ou acompanhantes e comunidade, transformando-os em co-participantes do processo de prevenção de LPs no paciente crítico.

Por fim, a mudança de decúbito deve ser valorizada e considerada como uma medida primordial uma vez que, de fato, pode prevenir as LPs. Com isso, será possível diminuir a quantidade e a complexidade das ações assistenciais dos profissionais de Enfermagem e, consequentemente, aliviar a sobrecarga de tarefas.

\section{Referências}

1. Moraes JT, et al. Conceito e classificação de lesão por pressão: atualização do National Pressure Ulcer Advisory Panel. Enferm. Cent. O. Min. 2016 mai./ ago.; 6(2):292-306.

2. Olkoski E, Assis GM. Aplicação de medidas de prevenção para úlceras por pressão pela equipe de Enfermagem antes e após uma campanha educativa. Esc Anna Nery. 2016 abr./jun.; 20(2):363-9.

3. Silva MRV, Dick NRM, Martini AC. Incidência de úlcera por pressão como indicador de qualidade na assistência de Enfermagem. Rev. Enferm. UFSM. 2012 mai./ago.; 2(2):38-48.

4. Araújo $A A$, Santos AG. Úlceras por pressão em pacientes internados em unidades de terapia intensiva: revisão integrativa da literatura. Ciência \& Saúde. 2016 jan./abr.; 9(1):38-48.

5. Oliveira E, Santos LGS, Almeida KSM. Ações de Enfermagem na prevenção das úlceras por pressão em uma Unidade de Terapia Intensiva. Rev. Digital EFDeportes. 2014 jun; 19(193).

6. Campanili TCGF, et al. Incidência de úlceras por pressão em pacientes de Unidade de Terapia Intensiva Cardiopneumológica. Rev. Esc. Enferm. USP. 2015 jun; 49(Esp):7-14.

7. Barbosa TP, Beccaria LM, Poletti NA. Avaliação do risco de úlcera por pressão em UTI e assistência preventiva de Enfermagem. Rev. Enferm. UERJ. 2014 mai./jun.; 22(3):353-8.

8. Rocha SCG, et al. Comparação das escalas de avaliação de risco de lesão por pressão. Rev. Bras. Pesq. Saúde. 2016 out./dez.; 18(4):143-51.

9. Ruiz JC. Abordaje y manejo de las heridas. Ciudad de Mexico: Intersistemas; 2013.

10. Palhares VC, Palhares-Neto AA. Prevalência e incidência de úlceras por pressão em uma Unidade de Terapia Intensiva. J. Nurs. UFPE. 2014 out; 8(Suppl.2):3647-53.

11. Gothardo ACLO, et al. Incidência de úlcera por pressão em pacientes internados em Unidade de Terapia Intensiva adulto. J Health Sci Inst. 2017; 35(4):252-6.

12. Baó $A C P$, et al. Quality indicators: tools for the management of best practices in Health. Rev Bras Enferm. 2019 mar./abr.; 72(2):360-6.

13. Machado JP, Martins ACM, Martins MS. Avaliação da qualidade do cuidado hospitalar no Brasil: uma revisão sistemática. Cad. Saúde Pública. 2013 jun; 29(6):1063-82.

14. Mattos RM, et al. Educação em saúde aos trabalhadores de Enfermagem e acompanhantes sobre prevenção e tratamento de lesões de pele em dois hospitais de Petrolina-PE. Interfaces. 2015 ju./dez.; 3(1):22-32.

15. Souza CT, Prado RT. A utilização da escala de Braden na UTI para prevenção da úlcera por pressão. Rev. EDUC-Faculdade de Duque de Caxias. 2016 jan./jun.; 3(1):31-50.

16. Otto C, et al. Fatores de risco para o desenvolvimento de lesão por pres- são em pacientes críticos. Enferm. Foco. 2019 fev; 10(1):7-11.

17. Prodanov CC, Freitas EC. Metodologia do trabalho científico: métodos e técnicas da pesquisa e do trabalho acadêmico. 2.ed. Novo Hamburgo: Universidade Feevale; 2013.

18. Sousa JR, et al. Prevenção de lesão por pressão em pacientes internados na unidade de terapia intensiva: um enfoque nas medidas preventivas. BJSCR. 2019 dez./fev,; 25(2):120-3.

19. Pereira MO, Ludvich SC, Omizzolo JAE. Segurança do paciente: prevenção de úlcera por pressão em Unidade de Terapia Intensiva. Rev. Inova Saúde. 2016 dez; 5(2):29-44.

20. Vasconcelos CMB, Caliri MHL. Ações de Enfermagem antes e após um protocolo de prevenção de lesões por pressão em terapia intensiva. Esc. Anna Nery. 2017 jan; 21(1):1-9.

21. Swafford K, Culpepper R, Dunn C. Use of a comprehensive program to reduce the incidence of hospital-acquired pressure ulcers in an intensive care unit. Am J Crit Care. 2016 mar; 25(2):152-5.

22. Albuquerque $A M$, et al. Avaliação e prevenção da úlcera por pressão pelos Enfermeiros de terapia intensiva: conhecimento e prática. Rev. Enferm. UFPE On-Line. 2014 fev; 8(2):229-39.

23. Mendonça $P K$, et al. Prevenção de lesão por pressão: ações prescritas por Enfermeiros de centros de terapia intensiva. Texto Contexto Enferm. $2018 \mathrm{abr} ; 27(4): 1-10$.

24. Sanches BO, et al. Adesão da Enfermagem ao protocolo de lesão por pressão em unidade de terapia intensiva. Arch. Health Sci. 2018 jul./dez.; 25(3):27-31.

25. Bauer K, et al. Pressure ulcers in the United States inpatient population from 2008 to 2012: results of a retrospective nationwide study. Ostomy Wound Manage, v.62, n.11, p.30-8, Nov. 2016.

26. Cruz JDA, et al. Prevenção de úlceras por pressão e segurança do paciente: percepções de Enfermeiros em terapia intensiva. Rev. Contexto \& Saúde. 2015 jan./jun.; 15(28):62-6.

27. Dantas ALM, et al. Prevenção de úlceras por pressão segundo a perspectiva do Enfermeiro intensivista. Rev. Enferm. UFPE On-Line. 2013 mar; 7(3):706-12.

28. Rolim JA, et al. Prevenção e tratamento de úlceras por pressão no cotidiano de Enfermeiros intensivistas. Rev Rene. 2013 jan./fev.; 14(1):148-57.

29. Gomes NP, et al. Prevenção de lesão por pressão: revisão integrativa da produção da Enfermagem brasileira. Rev. Ciência (In) Cena. 2017 mai; $1(5): 139-57$.

30. Menezes LCG, et al. Cuidados clínicos e gerenciais de Enfermagem na prevenção de úlcera por pressão. Rev. Estima. 2017 fev; 15(2):107-14.

31. Davis KG, Kotowski SE. Role of bed design and head-of-bed articulation on patient migration. J Nurs Care Qual. 2015 jul./sep.; 30(3):1-9. 